\title{
DESAFÍOS EN LA ENSEÑANZA DE LENGUAS INDÍGENAS: EL CASO DEL MAPUDUNGUN EN EL PROGRAMA DE EDUCACIÓN INTERCULTURAL BILINGÜE (PEIB) DE CHILE
}

\section{CHALLENGES IN TEACHING INDIGENOUS LANGUAGES: THE CASE OF MAPUDUNGUN IN THE BILINGUAL INTERCULTURAL EDUCATION PROGRAM (PEIB) OF CHILE}

\section{Cecilia Quintrileo Llancao* Elizabeth Quintrileo Llancao**}

\section{RESUMEN}

Las políticas lingüísticas del Estado chileno han privilegiado el monolingüismo, en detrimento del bilingüismo, de manera que las lenguas indígenas han sido sistemáticamente excluidas del ámbito educativo. Sin embargo, en los últimos años, se han implementado políticas de revitalización de las lenguas minoritarias, mediante la inclusión de las lenguas indígenas en curriculum escolar nacional, lo que implica un cambio de paradigma en relación con las políticas de planificación lingüística, como también la reconstrucción de identidades lingüísticas para sus hablantes. Una de las políticas de revitalización es el Programa Intercultural Bilingüe en Chile, el cual es analizado en este artículo enfocándose en sus orientaciones didácticas, y específicamente, en el caso del mapudungun, lengua hablada por el pueblo mapuche en Chile. Dicho análisis se lleva a cabo considerando algunos principios del enfoque comunicativo de enseñanza de lenguas (RICHARDS y RODGER, 2001; SAVIGNON, 2002) para posteriormente aportar con una serie de sugerencias metodológicas para la enseñanza de las lenguas indígenas. Entre las sugerencias metodológicas se destacan las necesidades del aprendiz y el diseño de actividades didácticas situadas en el contexto de aula comunicativa. Con respecto a las necesidades del aprendiz, se propone una metodología de base cooperativa, es decir, de todos los entes involucrados en el proceso formativo, considerando: a) las necesidades del alumno, y b) el diseño de actividades didácticas situadas en un contexto de 'aula comunicativa', con énfasis en el desarrollo de la competencia comunicativa. Desafortunadamente, la integración de este último actor en el sistema escolar se ha visto obstaculizada, por ende se interrumpe el desarrollo de la competencia comunicativa, por lo cual se hace necesaria la participación activa tanto de los educadores tradicionales como de la comunidad indígena, ya que, la comunicación es parte fundamental de la tradición oral del mundo indígena. Concretamente, se propone un levantamiento de metodologías basadas en la comunidad y con un enfoque colaborativo que

\footnotetext{
* Universidad Austral de Chile, Chile. cecilia.quintrileo@uach.cl

** Universidad de Bío Bío, Chile. equintrileo@ubiobio.cl
} 
apunten a cubrir las necesidades comunicativas del alumno, a través actividades didácticas situadas.

Palabras claves: políticas lingüísticas e identidad; interculturalidad; lengua Mapuche, educación intercultural.

\section{ABSTRACT}

The linguistic policies of the Chilean State have privileged monolingualism, to the detriment of bilingualism, so that indigenous languages have been systematically excluded from the educational field. However, in recent years, policies for the revitalization of minority languages have been implemented, through the inclusion of indigenous languages in the national school curriculum, which implies a paradigm shift in relation to language planning policies, as well as the reconstruction of linguistic identities for their speakers. One of the policies of revitalization is the Intercultural Bilingual Program in Chile, which is analyzed in this article focusing on its didactic orientations, and specifically, concentrating on the case of Mapudungun, a language spoken by the Mapuche people in Chile. This analysis is carried out considering some principles of the communicative approach of language teaching (RICHARDS \& RODGER, 2001, SAVIGNON, 2002) to later contribute with a series of methodological suggestions for the teaching of indigenous languages. Among the methodological suggestions are the needs of the learner and the design of didactic activities located in the context of a communicative classroom. Regarding the needs of the apprentice, a cooperative-based methodology is proposed, that is, of all the entities involved in the training process, considering: a) the needs of the student, and b) the design of didactic activities located in a context of 'communicative classroom', with emphasis on the development of communicative competence. Unfortunately, the integration of this last actor in the school system is hindered which inhibits the teaching of the indigenous language, as a result, the development of communicative competence is interrupted, for this reason, the active participation of both traditional educators and of the indigenous community is a fundamental part because of the oral tradition of the indigenous world. Finally, this work proposes community - based methodologies with a collaborative approach aimed at covering the communicative needs of the student through located educational activities.

Keywords: language policies and identity; interculturality; the Mapuche language; intercultural education.

\section{INTRODUCCIÓN}

A partir de los años noventa, la situación lingüística del mapudungun, lengua hablada por los mapuche en Chile y Argentina, ha sido caracterizada por su desplazamiento lingüístico, debido a una serie de circunstancias de subordinación política, económica y sociocultural (LAGOS, 2005). Otros autores han planteado el estado de amenaza del mapudungun, augurando su reemplazo progresivo por el castellano y alertando la posibilidad de convertir al pueblo mapuche en una comunidad monolingüe (VERGARA, GUNDERMANN Y FOERSTER, 2013).

De acuerdo a Salas (1992), los mapuche fueron forzados al bilingüismo, en tanto que los hispanos hablantes llegados al área de contacto, siguieron siendo 
monolingües del castellano, quedando inmersos en un mundo ajeno, en el cual no había ni hay lugar para ellos en cuanto a grupo socioculturalmente diferenciado. De este modo, las políticas lingüísticas del Estado chileno han privilegiado el monolingüismo en detrimento del bilingüismo, principalmente en ámbitos institucionales, como la administración y la enseñanza, donde las lenguas indígenas han sido excluidas históricamente. De este modo, desde el punto de vista político, las autoridades de turno se han empeñado en homogeneizar histórica, cultural e idiomáticamente a la nación chilena, y como consecuencia de ello, no es desconocida la prohibición factual de hablar la lengua mapuche en las escuelas, siendo sancionado su uso por los maestros (ÁLVAREZ-SANTULLANO y FORNO, 2008). Loncon (2013) plantea que los pueblos indígenas han permanecido en una situación de colonialidad, subordinación, y presión, circunstancia que ha repercutido en una condición desfavorable frente a la cultura nacional. Sin embargo, a pesar de las enormes presiones externas capaces de conducir hacia su total asimilación, Catrileo (2010) destaca la tenacidad en la utilización de la lengua mapuche y el valor que ésta tiene en el Mapu y en los rituales, lo que ha permitido su mantención hasta el siglo XXI. En consecuencia, se pone de manifiesto la resistencia frente una política lingüística favorable al monolingüismo y a una política de identidad que apunta a la asimilación cultural y étnica de los mapuche, es decir, que busca la erradicación de sus especificidades identitarias.

De acuerdo con estos y otros estudios, existe una realidad lingüística del mapudungun que merece debida atención y preocupación, con miras a acciones específicas orientadas a procesos de revitalización de esta lengua.

En el ámbito educativo, particularmente desde las políticas del Ministerio de Educación de Chile (MINEDUC), surge en 1996 el Programa de Educación Intercultural Bilingüe (PEIB), como resultado de la promulgación de la Ley Indígena $19.253^{1}$, que establece normas sobre protección fomento y desarrollo de los pueblos indígenas en Chile. Este programa entra en vigencia en forma gradual a partir del año 1996, haciéndose obligatoria su implementación a partir del año 2010 en establecimientos con matrícula indígena igual o superior al 50\%, y, en el presente, en aquellos con un porcentaje igual o mayor al $20 \%$ de estudiantes de ascendencia indígena, lo que, sin duda, ha implicado una transformación del curriculum escolar en Chile y un cambio de paradigma en relación con las políticas de planificación lingüística, particularmente con la enseñanza de lenguas indígenas en el país.

No obstante, si bien las políticas del MINEDUC han puesto atención en la enseñanza de las lenguas indígenas en Chile, la implementación del PEIB supone

1. Ley 19.253, llamada también "Ley Indígena", fue promulgada en el año 1993. 
una serie de desafíos en el sistema escolar vigente, por varias razones: a) El aumento en la cobertura que pretende abarcar el sector de lengua indígena en el contexto escolar en Chile, b) la carencia de metodologías y propuestas didácticas específicas y situadas a las necesidades de cada contexto y zona geográfica, c) la necesidad de formación de profesores en educación intercultural, de acuerdo a los distintos contextos y diversidad lingüística, y, finalmente escasas investigaciones o reportes de experiencias con respecto a metodologías de enseñanza de lenguas indígenas en Chile (QUINTRILEO y VALENZUELA, 2014; ESPINOZA, 2016).

El presente trabajo se centra en el marco curricular para la enseñanza de las lenguas indígenas, propuesto por el Ministerio de Educación (MINEDUC) del Gobierno de Chile, principalmente en las orientaciones didácticas del PEIB, considerando el caso del mapudungun, a fin de aportar a la discusión con respecto a acciones o microacciones de planificación lingüística y metodologías con miras a la revitalización de esta lengua. El objetivo es analizar tres orientaciones didácticas del PEIB: los estudiantes son el centro de los aprendizajes, la comunidad como recurso y fuente de saberes y el uso de nuevas tecnologías, desde un enfoque comunicativo de enseñanza de lenguas (RICHARDS y RODGERS, 2001; SAVIGNON, 2002), para posteriormente aportar con una serie de sugerencias metodológicas para la enseñanza de las lenguas indígenas en general, y del mapudungun, en particular.

El trabajo se estructura en dos apartados: el primero presenta el marco conceptual y metodológico; el segundo, se centra en el análisis y discusión de los resultados, para finalmente exponer las conclusiones.

\section{MARCO CONCEPTUAL Y METODOLÓGICO}

En este apartado, se describe en primer lugar el marco conceptual del estudio, pasando revista a algunos principios teóricos del enfoque comunicativo de enseñanza de lenguas; en segundo lugar, se describe el marco metodológico adoptado en este trabajo.

\subsection{El enfoque comunicativo de enseñanza de lenguas}

Un enfoque comunicativo de la lengua implica, en primer lugar, utilizar la lengua como medio de comunicación, y, en segundo lugar, lograr la competencia para precisamente usar la lengua de una determinada comunidad lingüística, de manera tal que logremos hacernos entender y ser entendidos. De acuerdo con este enfoque, la lengua es ante todo comunicación, por lo tanto, su objetivo de 
enseñanza es precisamente alcanzar la competencia comunicativa (SAVIGNON, 2002).

De acuerdo con Savignon (2002), el término 'competencia' se entiende, por una parte, como la expresión, interpretación y negociación de significado, y por otra, la habilidad de los estudiantes de lenguas para interactuar con otros hablantes. Savignon (1983), además, explica que la "competencia comunicativa" es la expresión, interpretación, y negociación de significado involucrando interacción entre dos o más personas, o entre una persona y un texto oral o escrito.

Richards y Rodgers (2001), por su parte, explican este enfoque desde dos puntos de vistas teóricos, el primer de ellos se relaciona con el lenguaje, en el cual se establece que éste es un medio de comunicación con las siguientes características:

1. El lenguaje es un sistema para la expresión de significados

2. La primera función del lenguaje es permitir la interacción y la comunicación

3. La estructura del lenguaje refleja su funcionalidad y usos comunicativos

4. Las unidades primarias del lenguaje no son solamente sus aspectos gramaticales y estructurales, sino son categorías de significados funcionales y comunicativos.

El segundo punto de vista se relaciona con el aprendizaje. Richards y Rodgers (2001) señalan que la enseñanza de lenguas a través de enfoque comunicativo se basa en tres principios:

1. Principio de Comunicación: Actividades de aprendizaje que promuevan la comunicación.

2. Principio de Tarea: Actividades en las cuales el lenguaje se usa para llevar a cabo tareas significativas o útiles para promover el aprendizaje.

3. Principio de Significado: El lenguaje significativo para el aprendiz apoya el proceso de aprendizaje.

Según Richards y Rodgers (2001), la Enseñanza Comunicativa de la Lengua no es un método sino un enfoque en lo cual estos tres principios se aplican siguiendo siempre una visión comunicativa de la lengua que presenta las siguientes características en el proceso de enseñanza - aprendizaje de una segunda lengua:

- Los alumnos aprenden un idioma usándolo para comunicarse.

- Las actividades del aula tienen que buscar una comunicación auténtica y significativa.

- La fluidez es una dimensión importante de la comunicación. 
- La comunicación supone la integración de diferentes habilidades lingüísticas.

- El aprendizaje es un proceso de construcción creativa que implica el ensayo y el error.

Siguiendo los lineamientos basados en el uso de la lengua con fines comunicativos, las distintas propuestas didácticas para la enseñanza de una lengua desde el enfoque comunicativo comparten el objetivo fundamental de enseñar a usar la lengua meta a través de actividades prácticas que permitan al alumno aprender y ejercitar la comunicación en el aula (CASSANY, 1999). Asimismo, sobre la base de los principios de comunicación, tarea y lenguaje significativo, este enfoque de enseñanza focaliza el acto de comunicación, para el desarrollo de la competencia comunicativa (SAVIGNON, 2002). Su esencia se encuentra en el enriquecimiento de los objetivos de aprendizaje, pues no se trata únicamente de que los estudiantes adquieran un determinado sistema lingüístico, sino de que sean capaces de utilizarlo para comunicarse de forma adecuada y efectiva, en situaciones reales (LITTLEWOOD, 1998).

\subsection{Marco metodológico}

Como ya se ha señalado, el presente estudio se centra en el marco curricular para la enseñanza de las lenguas indígenas, propuesto por el Ministerio de Educación (MINEDUC) del Gobierno de Chile, principalmente en las Orientaciones Didácticas del Programa de Educación Intercultural Bilingüe (PEIB) (MINISTERIO DE EDUCACIÓN, 2017), considerando el caso del mapudungun, como asignatura de lengua indígena, con el propósito de aportar a la discusión sobre microacciones de planificación lingüística y metodologías para la revitalización de esta lengua.

La investigación es de tipo cualitativa con alcance descriptivo, y el análisis planteado se desarrolla a la luz de principios del enfoque comunicativo de enseñanza de lenguas (RICHARDS y RODGERS, 2001; SAVIGNON, 2002), para aportar con una serie de sugerencias metodológicas para la enseñanza de las lenguas indígenas en general, y del mapudungun, en particular, considerando dos aspectos: a) las necesidades del alumno, y b) el diseño de actividades didácticas situadas en un contexto de 'aula comunicativa', con énfasis en el desarrollo de la competencia comunicativa.

\section{ANÁLISIS Y DISCUSIÓN DE LOS RESULTADOS}

El Programa de Educación Intercultural Bilingüe (PEIB) en Chile surge como política educativa el año 1996, producto de la promulgación de la Ley Indígena. 
Esta normativa crea la Corporación Nacional de Desarrollo Indígena (CONADI), la cual tiene entre otras misiones la de encargarse, en conjunto con el Ministerio de Educación, de promover planes de fomento de las culturas indígenas y desarrollo de un programa de educación intercultural bilingüe en áreas de alta densidad indígena, en el contexto de la diversidad cultural de pueblos indígenas presentes en el país y en virtud de las lenguas vernáculas con vitalidad lingüística: Aymara, Quechua, Mapuzungun y Rapa Nui (MINISTERIO DE EDUCACIÓN, 2011).

Entre sus principales lineamientos, los programas de estudio de Educación Intercultural Bilingüe establecen que Chile es un país multicultural y plurilingüe, donde convergen diversas culturas y lenguas, realidad que la escuela debiera acoger con el propósito de asegurar el acceso a oportunidades de aprendizaje de las lenguas indígenas. Los programas se articulan desde un enfoque de educación intercultural, con una doble orientación teórica, con énfasis en una perspectiva de bilingüismo aditivo, conjugada con un enfoque comunicativo-semántico de enseñanza de lengua. De esta manera, el MINEDUC destaca la finalidad de la educación intercultural, en términos de rescatar y valorar las diferencias existentes entre los grupos culturalmente diversos, y en donde las lenguas y culturas específicas adquieren gran relevancia, ya que busca el reconocimiento mutuo entre ellas (MINISTERIO DE EDUCACIÓN, 2011).

\subsection{Orientaciones didácticas}

Un análisis de las orientaciones didácticas contempladas en el PEIB, permite distinguir tres focos de atención: los estudiantes son el centro de los aprendizajes; la comunidad como recurso y fuente de saberes, y el uso de nuevas tecnologías.

\subsubsection{Los estudiantes son el centro de los aprendizajes}

La orientación didáctica centrada en las particularidades del estudiante es un pilar fundamental del enfoque comunicativo de enseñanza de lenguas. De esta manera, el MINEDUC recomienda considerar las experiencias personales, identidades, intereses, gustos y realidades de todos los estudiantes que comparten la sala de clases, favoreciendo el diálogo intercultural, con miras a aprender a valorar y apropiarse de conocimientos distintos a los propios (MINISTERIO DE EDUCACIÓN, 2011).

Aunque esta dimensión resulta central en un enfoque comunicativo, debemos señalar que el PEIB solamente considera la implementación de la asignatura de lengua indígena en el sistema escolar en aquellos establecimientos con matricula 
igual o superior al 20\%. Por tanto, el centro de los aprendizajes son los alumnos de ascendencia indígena y no indígenas de aquellos establecimientos que cumplan con el 20\% del alumnado contemplado por el PEIB. En los casos restantes, donde los establecimientos no presentan matrícula indígena acorde a los requisitos impuestos por el Ministerio de Educación, no es obligatoria la implementación del PEIB. No obstante, ca qué razones obedece este tipo de políticas, que fragmenta la educación intercultural? $\mathrm{Al}$ respecto, consideramos que todos los niños y jóvenes del país, sin excepción, deberían tener derecho a una educación intercultural bilingüe, y aprender mínimamente una lengua indígena, porque, de otro modo, es bastante discutible el desarrollo de sujetos interculturales a nivel país, de modo que se produzca una real trasformación social y valoración de las culturas indígenas en Chile.

\subsubsection{La comunidad como recurso y fuente de saberes}

En los programas de estudio se legitima la comunidad como fuente de saberes y de experiencia, valorando el conocimiento de las comunidades hablantes de la lengua originaria, especialmente la participación de la población de mayor en edad, conocimiento ancestral y experiencia cultural. De esta manera, reconoce las fuentes de conocimiento, y recomienda la consulta e interacción de los estudiantes con estas. Llama la atención la autoridad, como reconocimiento simbólico asignado por el PEIB a la comunidad lingüística local, particularmente la familia o entorno familiar de cada estudiante, como, por ejemplo, las personas que asumen el rol de abuelos en una familia, como fuente indiscutible del saber y conocimiento. Además, se hace hincapié en que la escuela por sí sola no puede asumir las tareas de recuperar una lengua y fortalecer la identidad, la autoestima y los valores de las culturas originarias:

Se recomienda incorporar a la familia, los sabios y otras autoridades tradicionales de la comunidad, ya que estos, como sujetos portadores de la cultura podrán aportar con sus vivencias, valores, consejos y saberes desde su mundo cultural y traspasar sus experiencias a las nuevas generaciones, por medio de los discursos, relatos, cuentos, cantos, bailes y expresiones artísticas, entre otras (MINISTERIO DE EDUCACIÓN, 2011, pp. 11-12).

Asimismo, se destaca la contribución de los educadores tradicionales en el proceso formativo, especialmente en el rescate y revitalización de las lenguas indígenas, aspecto que constituye un avance significativo en la visión de las políticas educativas desde el Ministerio de Educación.

Sin duda, esta orientación didáctica reviste especial importancia en el contexto de las políticas de planificación lingüística, no solo por incorporar la enseñanza de las lenguas indígenas en el curriculum escolar, promoviendo así el bilingüismo - en contraste con el reciente pasado histórico, que se caracteriza por 
políticas integracionistas, orientados más bien al monolingüismo - sino también por reconocer el rol de las autoridades tradicionales y de las comunidades culturales en materia de la revitalización de las lenguas indígenas.

\subsubsection{Uso de nuevas tecnologías}

Además de los puntos anteriores, los Programas de Estudio proponen el uso de nuevas tecnologías, ya sean digitales o de comunicación, en el trabajo didáctico del sector, considerando varias ventajas, como el otorgamiento de mayor estatus social a la lengua, y la posibilidad de usarla en los medios tecnológicos modernos. Igualmente, esta orientación didáctica resulta de interés en la enseñanza de lenguas indígenas, pues desde el punto de vista discursivo considera los diferentes ámbitos o canales de comunicación, tanto los tradicionales propios de la oralidad como la conversación y otras formas de interacción, así como la comunicación a través de medios digitales. De esta manera, se sitúa la enseñanza de las lenguas indígenas en la era de digitalización y globalización, considerando la proliferación de tecnologías digitales, que posibilitan la existencia de dispositivos masivos de comunicación, entretenimiento e información (DE MORAES, 2011). Esta orientación constituye un paso adelante en la didáctica de las lenguas indígenas, ya que propicia una visión situada igualmente en los nuevos contextos de producción y recepción del discurso, alineándose con aportes de corrientes teóricas como la lingüística textual, la sociolingüística y la pragmática, entre otras.

Sin duda, esta orientación didáctica puede tener como referencia igualmente el uso de las redes sociales, y otras plataformas digitales, donde se comparten recursos de aprendizaje. Hoy en día, es posible observar el incremento de interés por aprender lenguas indígenas como el mapudungun. En Internet, por ejemplo, algunos grupos en Facebook cuentan hoy en día con un número no menor de miembros, quienes comparten materiales (Gramáticas del mapudungun, diccionarios, manuales, etc.), y propuestas didácticas sobre frases útiles, saludos, números y otros paradigmas léxicos. Lo interesante de los grupos en Facebook es que los usuarios no solo pueden compartir recursos, sino que también interactúan digitalmente, haciendo contribuciones, consultas, pero también críticas y correcciones idiomáticas. Evidentemente, considerando el caso de los neohablantes de lenguas indígenas en Chile, existe el riesgo de errores en la transmisión cultural de una lengua; por ello, la necesidad de una formación sistemática en lengua indígena en la escuela, de manera que las redes sociales y otros dispositivos electrónicos sean un espacio complementario de interacción, como parte de la cultura digital. 
En conexión con el punto anterior, hay que considerar que los alumnos de hoy son nativos digitales, suben y bajan información, se conectan permanentemente, y hacen uso frecuente de un dispositivo celular. De este modo, considerando esta última orientación didáctica, se puede conjugar la escritura digital, con la cultura ancestral, además de la comunicación por mensaje de voz, en lengua indígena. Sin ir más lejos, esta práctica es más común de lo que imaginamos; y como parte de trabajo de campo, hemos observado la lealtad lingüística hacia la lengua materna en hablantes nativos y neohablantes, en zonas como Ralco en Alto Bío-Bío; en este caso, hacia el chedungun -variante del mapudungun, en distintos ámbitos de comunicación. Por consiguiente, el monolingüismo, engendrado por las políticas lingüísticas del Estado, se ve contrapuesto hoy a nuevos espacios, como la comunicación virtual, los cuales pueden considerarse prácticas de interacción espontánea; pero, a su vez, muestras de resistencia a políticas monoglósicas.

A modo de síntesis, cada una de las orientaciones didácticas del PEIB en Chile puede observarse en la siguiente figura:

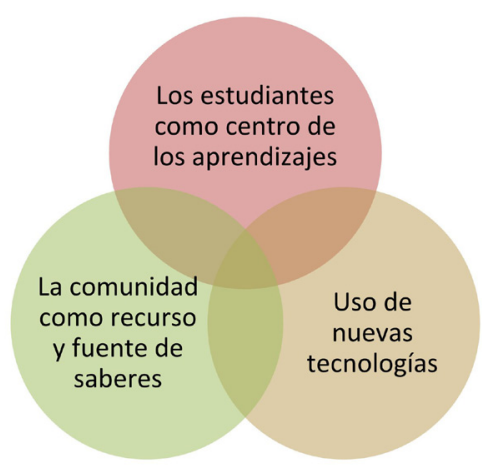

Figura1: Orientaciones didácticas del PEIB

Como se observa en la figura, las orientaciones didácticas del PEIB tienen como base el enfoque comunicativo de enseñanza de lenguas, el cual propone que la lengua es ante todo comunicación, es decir, la habilidad de usar la lengua en contextos sociales. Sin embargo, aun cuando el PEIB considera dicho enfoque, existen escasos reportes con respecto al desarrollo de la competencia comunicativa en lenguas indígenas a partir de la implementación del PEIB en escolares chilenos, por lo cual, resulta una incertidumbre el impacto real de este programa en el contexto escolar chileno. 
Otra gran interrogante es cómo articular una propuesta metodológica que integre cada una de las Orientaciones didácticas del PEIB, poniendo énfasis en las habilidades comunicativas relevantes para la enseñanza de las lenguas indígenas en la escuela, debido a su tradición oral. Téngase presente que la oralidad constituye una forma de transmisión del conocimiento ancestral el que se expresa y se enriquece de generación en generación. No es un conocimiento estático, sino que se adapta e incluye nuevos elementos a partir de los cambios contextuales y de la colaboración de sus participantes a través del tiempo. En las Bases Curriculares, el eje de Oralidad se divide en Tradición Oral y en Comunicación Oral, lo que debe ser considerado de manera conjunta (MINISTERIO DE EDUCACIÓN, 2011). La Tradición Oral es parte del patrimonio inmaterial de los pueblos originarios, y está estrechamente vinculada al reconocimiento y valoración del acto de escuchar. La comunicación oral tiene por finalidad fomentar la práctica interaccional del idioma. Para tal propósito, es necesario adoptar un enfoque en el cual se estudie, enseñe y se utilice la lengua desde un punto de vista comunicativo.

Para finalizar el análisis que nos ocupa, y con el propósito de complementar la discusión, presentamos una serie de sugerencias metodológicas para la enseñanza de las lenguas indígenas en general, y del mapudungun, en particular, centrándonos en dos aspectos: las necesidades del alumno aprendiz y el diseño de actividades didácticas situadas en un contexto de 'aula comunicativa', con énfasis en el desarrollo de la competencia comunicativa.

\subsection{Hacia una metodología de enseñanza de lengua indígena en contexto intercultural: sugerencias metodológicas}

Si se consideran las Orientaciones Didácticas del PEIB, la interrogante es ćcómo articular una propuesta metodológica que integre cada uno de estos aspectos (los estudiantes como centro de aprendizajes, la comunidad como recurso y fuente de saberes, y el uso de nuevas tecnologías)? Como ya hemos planteado, para discutir este punto, a continuación, esbozamos una serie de sugerencias metodológicas, considerando: a) las necesidades del alumno, y b) el diseño de actividades didácticas situadas en un contexto de 'aula comunicativa', con énfasis en el desarrollo de la competencia comunicativa.

\subsubsection{Adecuarse a las necesidades del alumno}

La orientación didáctica centrada en las particularidades del estudiante, como centro de los aprendizajes, incide en el cumplimiento de los objetivos y 
contenidos que se imparten. De este modo, el diseño didáctico debe orientarse hacia los requerimientos, como también intereses y metas de cada alumno. En una palabra, podría proponerse una metodología de base cooperativa (RICHTERICH, 1985), lo que en el PEIB se traduciría en la participación conjunta de todos los entes involucrados en el proceso formativo: el alumno y la dupla pedagógica, integrada por el educador tradicional y el profesor mentor. Debido a la escasez de profesores en educación intercultural en Chile, el sistema ha recurrido a la figura del Educador tradicional, portador del conocimiento ancestral, de la lengua y la cultura indígenas; sin embargo, la integración de este actor en el sistema escolar ha sido en muchos casos obstaculizada, porque no se ha orientado debidamente la función pedagógica de este último, lo que ha llevado, en algunos casos, al fracaso de la figura de la dupla pedagógica (LONCON, 2013; ESPIÑOZA, 2016), Sin embargo, como hemos reiterado, de acuerdo a algunas observaciones en trabajo de campo, existen también experiencias destacadas al respecto, particularmente en zona de Alto Bío-Bío.

En Chile, cada establecimiento diseña su propio modelo educativo, sin embargo, existen muy pocos reportes y evidencias sobre la incorporación del alumnado en un modelo educativo, donde se haya incorporado el PEIB. En un enfoque comunicativo, el docente asume un rol de mediador de los contenidos, y en el caso del PEIB, la figura del Educador Tradicional es central como mediador de la lengua y la cultura. Por tanto, se requiere la necesidad de formación continua, capacitaciones tanto para el Educador como para el Profesor Mentor, con miras a optimizar los procesos formativos y curriculares.

Siguiendo una perspectiva de modelo cooperativo centrado en el desarrollo de la competencia comunicativa, y atendiendo a las especificidades del contenido cultural, deberíamos preguntarnos: por una parte, ¿̇cómo facilitar la mediación del contenido cultural?, y, por otra parte, ¿cómo quiere aprender el alumno? En este punto, el diseño didáctico puede atender a las necesidades comunicativas de los estudiantes, conjugando las habilidades (comunicación oral y escritura, comprensión oral, etc.), los requerimientos socioculturales (temas de interés, géneros discursivos diversos tales como la conversación en todas sus formas), y preferencias didácticas como estilos de aprendizajes, metodologías y actividades de interés. Loncon (2013) plantea que los Educadores Tradicionales emplean variedad de métodos, como el tradicional, ancestral, gramatical en el aula, y al respecto, sugiere conjugar los métodos más exitosos como el enfoque comunicativo, sin dejar de lado la especificidad de la transmisión oral. En esta misma línea, consideramos la pertinencia y necesidad de nuevas propuestas y diseños didácticos, aportados por la propia comunidad, considerando las experiencias de los educadores tradicionales. En este 
sentido se hace necesaria una pedagogía basada en el lugar, la comunidad y la cultura (REYHNER y KUMAR, 2015) que potencie y reconozca los conocimientos de los sabios de las distintas comunidades indígenas considerando sus particularidades tanto lingüísticas como culturales.

Por lo tanto, un modelo de base cooperativa debería preguntarse ćpara qué quiere el alumno aprender? Es decir, prestar atención a las eventuales metas de los niños y jóvenes, lo que permitiría avanzar en la toma de conciencia de los procesos de motivación de los propios alumnos. Este último punto tiene especial importancia por sus implicancias a nivel de las actitudes que manifiestan los hablantes y no hablantes de una lengua indígena, tomando en consideración la escasa valoración de estas lenguas, como resultado de circunstancias históricas, de políticas públicas asimilacionistas y de ideologías etnocentristas y racistas. De manera que una clara visión, conocimiento de las metas o motivaciones de logro de los alumnos podría contribuir incluso a revertir actitudes de rechazo hacia metodologías, estilos y contenidos culturales específicos.

Finalmente, un enfoque centrado en las necesidades del alumno debería conocer y atender a su perfil sociolingüístico, de acuerdo a cada nivel y contexto geográfico. En Chile, el desafío es enorme, pues no contamos hoy con instrumentos claros para levantar diagnósticos sobre la competencia comunicativa de los niños y jóvenes; y evidentemente, un modelo con un enfoque comunicativo debería atender a la diversidad lingüística, particularmente en el caso del mapudungun.

\subsubsection{Diseñar actividades didácticas situadas en un 'aula comunicativa', con énfasis} en el desarrollo de la competencia comunicativa.

Siguiendo los lineamientos del enfoque comunicativo, consideramos que la finalidad del proceso formativo es la competencia comunicativa. De esta manera, los diseños didácticos deben contemplar el desarrollo de actividades, centradas tanto en aspectos del sistema lingüístico, como también en su utilización en situaciones concretas. Por tanto, la idea es conjugar el conocimiento de los aspectos gramaticales (fonéticos, morfológicos y léxicos), propios de una lengua indígena específica, para la elaboración de enunciados y discursos.

Por ejemplo, como sugerencia metodológica, puede enseñarse el uso de determinantes específicos a través de diálogos breves, como es el caso de los demostrativos, y otros deícticos, cuya interpretación requiere de los elementos del contexto. Por ejemplo: Pregunta: Chem tïfa? (Qué es esto), Respuesta: Tüfa lifru (esto es un libro). Asimismo, sugerimos el diseño didáctico orientado a situaciones concretas, como el destacado caso de los pronombres en mapudungun - lengua que 
presenta número singular, pero igualmente pronombres de número dual (Inchiw, eymu, feyengu), y número plural (Iñchiñ, eymün, fechengün). Cada uno de estas particularidades gramaticales puede enseñarse igualmente a través del uso de diálogos o actos de habla específicos como los saludos (pentukum), poniendo así la gramática en uso. ${ }^{2}$ Del mismo modo, a nivel léxico, campos semánticos específicos en relación con el ser humano en su entorno familiar, la tierra, como hábitat natural, los rituales, etc. ${ }^{3}$ Evidentemente, por límites de espacio, no es posible proporcionar una lista acabada de los aspectos gramaticales y léxicos propios de las lenguas indígenas contempladas en el PEIB.

En segundo lugar, sugerimos promover actividades con tareas o propósitos específicos, con énfasis en la producción discursiva. Con ello, ubicamos el discurso en el centro del proceso de aprendizaje, como máxima unidad de comunicación. Precisamente, en los programas de séptimo año del PEIB, se focalizan diferentes tipos de discurso, como, por ejemplo, los 'epew' - relato tradicional oral de la cultura mapuche -, los cuales no solo responden a una superestructura textual determinada, sino que además dan cuenta de la transmisión de valores específicos, culturalmente determinados por la tradición oral y ancestral propia del mundo indígena.

A la par de la transmisión oral, sugerimos ampliar el estudio de géneros discursivos, en congruencia con el contexto sociopolítico imperante. Por ejemplo, el discurso público mapuche aparece invisibilizado en los programas del PEIB, más concretamente, las grandes demandas de los pueblos indígenas, como los derechos lingüísticos o el reconocimiento constitucional como pueblos, constituyen temas pendientes, cuya ausencia devela una política de desvalorización de las identidades indígenas. De otro modo, ćcuáles serían las razones para excluir el conocimiento del discurso público configurado por intelectuales y políticos mapuche, quienes han contribuido al diálogo y al debate sobre materia indígena en el país?

Igualmente, sugerimos enfatizar contenidos orientados hacia la producción textual, lo que resulta propicio, pues permite fomentar tanto la tradición oral como la escritura creativa; por ejemplo, a través de poemas, canciones, etc., propios de la cultura ancestral, como también sobre la base de la ampliación de un corpus literario, relevando la poesía de reconocidos y connotados poetas mapuche, como Elicura Chihuailaf, Leonel Lienlaf o Graciela Huinao, entre otros, vivos exponentes de la lengua y cultura mapuche. Entre las ventajas de la creación poética, se encuentran el

2. Los ejemplos aquí presentados se encuentran en Catrileo (1987): Mapudunguyu. Curso de Lengua Mapuche.

3. Una interesante herramienta teórica para el trabajo de campos semánticos es el Diccionario Lingüístico Etnográfico de la Lengua Mapuche. Mapudungun, Español, Inglés. (Catrileo, 2017). 
desarrollo de la escritura, la expresión oral creativa, el incremento de la expresividad verbal, mediante los recursos de la retórica y oratoria. Además, la tradición literaria contribuye al prestigio y estatus de una lengua y, en el caso particular del PEIB, al reconocimiento de saberes, propios del mundo indígena. El diseño de actividades didácticas de esta naturaleza contribuye igualmente al bilingüismo, mapudungun y español, y puede constituir una práctica de valorización de la identidad mapuche, la cual no se restringe a un conocimiento meramente ancestral, sino que comporta un tipo conocimiento complejo, dinámico y multidimensional, lo que se evidencia en la producción literaria, y científica, aportada por prestigiosos intelectuales, artistas, y creadores mapuche.

Por tanto, las nuevas prácticas discursivas, literarias y no literarias, en distintos soportes - oral, audiovisual, escrito - dan cuenta de un nuevo escenario para las lenguas indígenas, donde sus usuarios no tienen el fantasma de la presión política, que ha excluido sistemáticamente a los pueblos indígenas del espacio público, de la administración, de la educación, y de la política.

\section{CONCLUSIONES}

El análisis del PEIB, específicamente de sus tres Orientaciones Didácticas (los estudiantes son el centro de los aprendizajes; la comunidad como recurso y fuente de saberes, y el uso de nuevas tecnologías), ha permitido discutir la necesidad de articular estas tres dimensiones, a través de diseños didácticos, que conjuguen metodologías del enfoque comunicativo, como también nuevas propuestas de parte de la propia comunidad ancestral.

De modo concreto, se propone el levantamiento de metodologías basadas en la comunidad y, a su vez, con un enfoque colaborativo-profesor mentor, educador tradicional-alumno-que apunten a las necesidades comunicativas del alumno y a actividades didácticas situadas, lo que permitiría orientar una propuesta metodológica para diseñar un modelo de enseñanza que apunte al desarrollo de la competencia comunicativa en mapudungun.

El análisis realizado intenta contribuir a la discusión para el mejoramiento de los procesos de enseñanza de la lengua y sus revitalización. Los resultados arrojados por el análisis revelan que, si bien el PEIB en Chile cuenta con fortalezas, es necesario, y de forma urgente, continuar mejorando su implementación, dada la situación de desplazamiento lingüístico, el cual, de forma permanente, amenaza a la lengua mapuche. El mapudungun, en su situación de lengua en riesgo requiere la unión de todos los esfuerzos necesarios de modo de incrementar sus niveles de 
reversión lingüística y lograr una mayor competencia comunicativa en este idioma. A través del análisis sobre las orientaciones del PEIB, se dispone de antecedentes para proponer líneas de acción específicas en relación con el diseño de actividades y material didáctico en contexto escolar, teniendo en consideración que los educadores tradicionales no sólo deben responder a los objetivos de aprendizaje de la asignatura de lengua indígena; antes bien, el concurso activo de ellos beneficiará las acciones encaminadas a la defensa y promoción del legado amenazado (FLORES y CÓRDOVA, 2012). Por esta razón, las debilidades detectadas y la puesta en marcha de nuevas propuestas para superarlas considerando un trabajo basado en la comunidad, constituyen el próximo paso en los esfuerzos de revitalización del mapudungun en Chile.

De igual forma, las necesidades del alumno, en este caso, de los niños y jóvenes mapuche, pueden aportar significativamente desde su perspectiva como miembros de las comunidades indígenas en lo concerniente a los aspectos más relevantes de la cultura mapuche y que no deben ser exceptuados en ningún programa de aprendizaje.

Asimismo, las orientaciones para un aula comunicativa deben, ante todo, responder tanto la tradición oral de la cultura, como a las manifestaciones iniciadas por intelectuales que aportan en la obra poética, el arte y el discurso escrito. Dichas manifestaciones, junto a la participación de distintos actores de la sociedad mapuche, visibilizan, promueven y fortalecen el desarrollo de lenguas y culturas, en ocasiones, altamente recesivas y estigmatizadas contribuyendo a una transformación positiva y efectiva de las ideologías y prácticas negativas hacia la diversidad lingüística y cultural (FLORES y CÓRDOVA, 2012).

La cultura mapuche, al igual que todas las culturas, es dinámica y creativa, y no responde a formas estáticas o estereotipos, por lo cual, la implementación de los programas de educación tanto en la lengua mapuche como otras lenguas indígenas, deben considerar las necesidades del alumno, así como también el conocimiento de las comunidades, y cuyo objetivo principal debe ser el logro de la competencia comunicativa como acción fundamental a favor del mantenimiento y reversión del desplazamiento de la lengua.

Por último, debemos señalar que la Declaración de las Naciones Unidades sobre los Derechos de los Pueblos Indígenas, aprobada el 13 de septiembre de 2008, afirma que todos los pueblos contribuyen a la diversidad y la riqueza de las civilizaciones y las culturas, que constituyen el patrimonio común de la humanidad. De igual forma, la Convención para la Salvaguardia del Patrimonio Cultural Inmaterial, adoptada el 17 de octubre de 2003, reconoce que las comunidades, en 
especial las indígenas, los grupos, y en algunos casos los individuos, desempeñan un importante papel en la producción, la salvaguardia, el mantenimiento y la recreación del patrimonio cultural inmaterial, contribuyendo con ello a enriquecer la diversidad cultural y la creatividad humana.

Por lo tanto, se hace evidente el aporte que cada cultura hace para conservar el bien de la humanidad, por lo cual, es necesario llevar a cabo acciones que contribuyan a revertir la situación de amenaza que actualmente afecta a las lenguas indígenas.

\section{REFERENCIAS}

ÁLVAREZ-SANTULLANO, P.; FORNO, A. (2008). La inserción de la lengua mapuche en el curriculum de escuelas con educación intercultural: un problema más que metodológico ALPHA, 26, pp. 9-28.

CATRILEO, M. (1987): Mapudunguyu. Curso de Lengua Mapuche. Valdivia: Universidad Austral de Chile.

CATRILEO, M. (2010). La Lengua Mapuche en el Siglo XXI. Valdivia: Facultad de Filosofía y Humanidades, Universidad Austral de Chile.

CATRILEO, M. (2017) Diccionario Lingüístico Etnográfico de la Lengua Mapuche. Mapudungun, Español, Inglés. Valdivia: Universidad Austral de Chile.

CASSANY, D. (1999). Los enfoques comunicativos: elogio y crítica: Lingüística y literatura, 36-37, pp. 11-33.

DE MORAES, D. (2011). La cruzada de los medios en América Latina. Gobiernos progresistas y políticas de comunicación. Buenos Aires: Paidós.

DECLARACIÓN DE LAS NACIONES UNIDAS SOBRE LOS DERECHOS DE LOS PUEBLOS INDÍGENAS. (2008). Publicado por las Naciones Unidas en Marzo de 2008.

ESPINOZA, M. (2016). Contextos, metodologías y duplas pedagógicas en el Programa de Educación Intercultural Bilingüe en Chile: una evaluación crítica del estado del debate. PEL, Pensamiento educativo. Revista de investigación educacional Latinoamericana, 53 (1), pp. 1-16. 
FLORES, J. y CÓRDOVA, L. (2012). Guía de Revitalización Lingüística: Para una gestión formada e informada. Ciudad de México, D.F: Centro de Investigaciones y Estudios Superiores de Antropología.

\section{GOBIERNO DE CHILE, MINISTERIO DE PLANIFICACIÓN Y COOPERACIÓN} (1998). Ley Indígena $N^{\circ}$ 19.253. Disponible en: https://www.leychile.cl/ Navegar? idNorma $=30620$. Último acceso: $12 / 10 / 2018$.

LAGOS, C. (2005). La vitalidad lingüística del mapudungun en Santiago de Chile, sus factores determinantes y consecuencias socioculturales: estudio exploratorio desde una perspectiva socio y etnolingüística Werken 006, pp. 23-37.

LITTLEWOOD, W. (1998). La enseñanza comunicativa de idiomas. Introducción al enfoque comunicativo. Madrid: Cambridge University Press.

LONCON. E. (2013). La importancia del enfoque intercultural y de la enseñanza de las lenguas indígenas en la educación chilena Revista Docencia, 51, pp. 44:51.

MINISTERIO DE EDUCACIÓN (2011). Programa de Educación Intercultural Bilingüe. Sector Lengua Indígena. Programa de Estudio Primer Año Básico. Sector Lengua Indígena Mapuzugun. Disponible en http://www.curriculumnacional.cl/inicio/ lengua-indigena/. Último acceso: 12/10/2018.

QUINTRILEO, C.; VALENZUELA, C. (2014). Competencia comunicativa en el programa intercultural bilingüe en el sistema educacional chileno. Calidoscópio, Vol. 12, n. 1, pp. 32-38.

REYHNER, J.; KUMAR, S. (2015). Overcoming the Legacy of Assimilationist Schooling. In: Reyhner, J. (org.) Teaching Indigenous Students, Honoring Place, Community, and Culture. University of Oklahoma Press, Norman Publishing Division of the University, pp. 11-35.

RICHARDS, C.; RODGERS, T. (2001). Task Based Language Teaching. Approaches and Methods in Language Teaching. In: Richards, J. y Rodgers, T. Cambridge University Press.

RICHTERICH, R. (1985). Besoins langagiers et objetives d'apprentissage. París: Hachette.

SALAS, A. (1992). El mapuche o araucano. Fonología, gramática y antología de cuentos. Madrid: Fundación MAPFRE AMERICA. Colecciones MAPFRE 1492. Colección Idioma e Iberoamérica. 
SAVIGNON, S. (1983). Communicative Competence: Theory and Classroom Practice: Reading, Massachusetts: Addison - Wesley Publishing Company.

SAVIGNON, S. (2002). Interpreting language teaching. London: Yale University Press New Heaven.

VERGARA, J.; GUNDERMANN, H.; FOERSTER, R. (2013). Estado, Conflicto Étnico y Cultura. Estudios sobre pueblos indígenas en Chile. Antofagasta: Universidad Católica del Norte - Chile.

Recebido: $17 / 08 / 2018$

Aceito: 20/10/2018 